\title{
LA MISE EN SYSTÈME ET L'ÉCONOMICISATION DE L'ENSEIGNEMENT EN FRANCE AU DÉBUT DES ANNÉES 1950 : LA FONCTIONNALISATION D'UNE INSTITUTION
}

\section{Philippe Bongrand}

De Boeck Supérieur | «Politix»

2012/2 n 98 | pages 35 à 56

ISSN 0295-2319

ISBN 9782804174279

Article disponible en ligne à l'adresse :

https://www.cairn.info/revue-politix-2012-2-page-35.htm

Distribution électronique Cairn.info pour De Boeck Supérieur.

(C) De Boeck Supérieur. Tous droits réservés pour tous pays.

La reproduction ou représentation de cet article, notamment par photocopie, n'est autorisée que dans les limites des conditions générales d'utilisation du site ou, le cas échéant, des conditions générales de la licence souscrite par votre établissement. Toute autre reproduction ou représentation, en tout ou partie, sous quelque forme et de quelque manière que ce soit, est interdite sauf accord préalable et écrit de l'éditeur, en dehors des cas prévus par la législation en vigueur en France. Il est précisé que son stockage dans une base de données est également interdit. 


\title{
La mise en système et l'économicisation de l'enseignement en France au début des années 1950 : la fonctionnalisation d'une institution
}

\author{
Philippe Bongrand
}

\begin{abstract}
Résumé - Alors que l'enseignement de la III République visait implicitement à reproduire les structures socio-économiques qui lui étaient contemporaines, pendant les "Trente glorieuses », au contraire, l'unification du système éducatif est explicitement conçue pour orienter des individus vers différentes catégories professionnelles à faire advenir. Tout en revendiquant l'objectif d'égalité des chances des enfants, " la réforme de l'enseignement » entend en particulier répondre à des besoins en cadres et techniciens. Ce projet d'une école aussi juste socialement qu'efficace économiquement est alors scellé dans les structures, durables, d'un système éducatif qui entend répartir les élèves vers les différents secteurs et niveaux professionnels. Observé entre la fin des années 1940 et le milieu des années 1950, au prisme de publications professionnelles et d'archives administratives, cet endossement d'une finalité économique se place au croisement, d'une part, de l'investissement accru des milieux agricoles et industriels dans la formation et, d'autre part, de concurrences internes à l'institution scolaire pour définir la réforme. Ce croisement est catalysé par la circulation de savoirs qui étayent et rendent compatibles des lectures économiques et démocratiques de l'enseignement. L'article envisage ainsi la politique publique de réforme de l'enseignement comme rouage d'un processus de fonctionnalisation économique de l'institution scolaire.
\end{abstract}


"La clé de la prospérité matérielle, c'est l'équipement intellectuel. La base de l'équipement intellectuel, c'est l'école ${ }^{1}$."

$\Upsilon$ Travailler à l'école pour réussir socialement ${ }^{2}$, justifier des frais d'inscription par le rendement financier des formations ${ }^{3}$, promouvoir buent aujourd'hui une fonction économique à l'enseignement. Ces usages ne sont pas dénués de bonnes raisons : les statistiques de « la relation formationemploi » montrent que le diplôme prémunit contre le chômage ${ }^{5}$; l'économie de l'éducation convainc que l'enseignement a la dignité d'un investissement ${ }^{6}$; l'idéologie méritocratique argumente l'idée que le lien entre réussites scolaire et sociale, malgré les faiblesses de sa mise en œuvre, offre un idéal légitime ${ }^{7}$. Ces usages ne vont pas non plus sans controverse : les manières pour l'enseignement de servir l'économie restent discutées ${ }^{8}$; des acteurs subissent l'orientation scolaire vers le monde du travail sans en reconnaittre la légitimité ${ }^{9}$; le principe même d'une utilité économique, qui contrevient aux définitions classiques de l'éducation en termes de désintéressement, est également dénoncé ${ }^{10}$. Cependant, par-delà des formes et modalités diverses, ces comportements et croyances expriment un phénomène fortement institué : la fonction économique de l'enseignement. Ils sont en cela interprétables comme des spécifications, parmi d'autres, d'un processus plus général qui conduit des agents à reconnaître - en même temps qu'à vouloir maîtriser - des effets économiques de l'enseignement. Pour interroger ce processus de fonctionnalisation ${ }^{11}$, dimension particulière de

1. Brochure du ministère de l'Éducation nationale « Le problème scolaire ? Le voici », Paris, SEVPEN, 1959, p. 15, conservée à la Bibliothèque nationale de France sous la cote 8 - LF244 - 216.

2. Millet (M.), Moreau (M.), dir., La société des diplômes, Paris, La Dispute, 2011.

3. Casta (A.), "Frais d'inscription et prêts étudiants en Angleterre : discours, origines et effets ", L'homme et la société, 4, 2010.

4. Garcia (S.), «Réformes de Bologne et économicisation de l'enseignement supérieur », Revue du MAUSS, $1,2009$.

5. Mazari (Z.), Meyer (V.), Rouaud (P.), Ryk (F.), Winnicki (P.), « Le diplôme : un atout gagnant pour les jeunes face à la crise », Bref du CEREQ, 283, 2011.

6. Gurgand (M.), Économie de l'éducation, Paris, La Découverte, 2005.

7. Tenret (É.), L'école et la méritocratie. Représentations sociales et socialisation scolaire, Paris, Presses universitaires de France, 2011.

8. Nussbaum (M. C.), Not for Profit: Why Democracy Needs the Humanities, Princeton, Princeton University Press, 2010.

9. Willis (P.), L'école des ouvriers. Comment les enfants d'ouvriers obtiennent des boulots d'ouvriers, Marseille, Agone, 2011 [1 $1^{\text {re }}$ éd. 1977].

10. Jones (K.), dir., L'école en Europe. Politiques néolibérales et résistances collectives, Paris, La Dispute, 2011 et Laval (C.), L'école n'est pas une entreprise. Le néolibéralisme à l’assaut de l'enseignement public, Paris, La Découverte, 2004.

11. "Des multiples relations qui s'établissent entre tous les agents ayant "à faire avec" l'institution, ou simplement affaire à elle, résulte son assimilation à une "fonction sociale" présumée indispensable... ", Lacroix (B.), Lagroye (J.), dir., Le président de la République. Usages et genèses d’une institution, Paris, Presses 
l'institutionnalisation de l'école, l'article retrace plus précisément la présence croissante d'acteurs et justifications économiques dans la réforme de l'organisation qui tend à monopoliser l'enseignement : le système éducatif.

C'est au cours des trente années qui suivent la Libération que l'organisation publique de l'enseignement prend, en France, la forme spécifique d'un système. Pour gouverner l'accroissement spectaculaire des effectifs et du taux de scolarisation, entre 1944 et 1968, la politique de « réforme de l'enseignement » modifie l'identité et l'agencement des différentes catégories d'établissements. À une juxtaposition de filières parallèles et ségrégées, elle entend substituer un ensemble d'établissements, harmonisés et coordonnés, capable d'alimenter et de canaliser des flux d'élèves suivant ses propres critères et objectifs. Cette " mise en système » - suivant une expression usuelle de l'historiographie ${ }^{12}$ - tend à ordonner rationnellement les établissements les uns par rapport aux autres, et, ensemble, relativement à des finalités. L'élaboration et la mise en œuvre de ce projet planificateur ${ }^{13}$ sont à l'origine de la situation actuelle :

« Maintenant que nous pouvons embrasser le XXe siècle dans son ensemble, les années 1959-1968 prennent un relief nouveau :l'Éducation nationale en sort transformée dans tous ses aspects. [...] Il y a là un grand tournant, comme une re-fondation à laquelle on n'a peut-être pas assez rendu justice et qui lui donne en tout cas pour l'essentiel le visage qu'elle présente encore au début du XXI' siècle $^{14}$. »

Si la décennie 1959-1968 présente en effet une accumulation spectaculaire de textes réglementaires, ce grand tournant s'engage dès les années qui précèdent, irréductibles à des rêves, non-décisions et échecs certes retentissants ${ }^{15}$. Des mesures de gestion apparemment anodines facilitent alors significativement l'unification des filières; des catégories durables d'experts légitimes de la chose scolaire y apparaissent ; le consensus sur l'accroissement inéluctable de la scolarisation post-obligatoire s'y noue : sous la IV République, comme le confirme ici l'enquête centrée sur le début des années 1950, la transformation du rôle social de l'école est amorcée ${ }^{16}$.

\footnotetext{
de la Fondation nationale des sciences politiques, 1992, p. 11 et Lagroye (J.), Offerlé (M.), dir., Sociologie de l'institution, Paris, Belin, 2010.

12. Lelièvre (C.), Histoire des institutions scolaires. 1789-1989, Paris, Nathan, 1990. C’est au milieu des années 1970 que les discours de politique scolaire désignent l'offre globale d'enseignement comme un "système ». 13. Berthelot (J.-M.), Le piège scolaire, Paris, Presses universitaires de France, 1983.

14. Prost (A.), Histoire de l'enseignement et de l'éducation en France. L'école et la famille dans une société en mutation (depuis 1930), Paris, Perrin, 2004, vol. 4, p. IV.

15. Cf. respectivement Robert (A.), L'École en France de 1945 à nos jours, Grenoble, Presses universitaires de Grenoble, 2010 ; Sadoun (M.), Donegani (J.-M.), « La réforme de l'enseignement secondaire en France depuis 1945. Analyse d'une non-décision ", Revue française de science politique, 26 (6), 1976 ; Dreyfus (F.-G.), " Un groupe de pression en action. Les syndicats universitaires devant le projet Billières de réforme de l'enseignement (1955-1959) », Revue française de science politique, 15 (2), 1965.

16. Chapoulie (J.-M.), «Une révolution dans l'école sous la Quatrième République ? ", Revue d'histoire moderne et contemporaine, 54 (4), 2007.
} 
Cette transformation se donne à voir, avant tout, sous l'angle de la " démocratisation ${ }^{17}$ ». Dans la continuité des projets qui, depuis le début du siècle, réclament l' " école unique ${ }^{18}$ ", la réforme prétend en effet rapprocher des filières afin d'y orienter les enfants non plus en fonction de leur origine sociale, mais, de manière rationnelle et juste, suivant leurs « goûts et aptitudes ${ }^{19}$ ». Les enjeux de justice sociale donnent ainsi un étalon central pour comprendre et évaluer cette politique ${ }^{20}$. Mais cet objectif postule que l'enseignement pèse sur la distribution des individus vers des positions sociales inégales : en mettant en continuité carrières scolaires et carrières socio-professionnelles, la " démocratisation » est ainsi indissociable de l'économicisation de l'institution scolaire. De fait, la réforme tente d'ajuster les structures et les modes de fonctionnement du service public d'enseignement à l'évolution anticipée des besoins en personnels qualifiés. C'est dans cette perspective que le décret Berthoin du 6 janvier 1959, coup d'envoi officiel de la réforme, exhorte à « investir à plein profit » dans l'école, présentée comme un instrument susceptible de convertir « l'expansion humaine " du baby-boom en " expansion économique ${ }^{21} »$. Ce texte majeur atteste ainsi une économicisation de l'enseignement - ou, plus précisément, une fonctionnalisation économique de l'organisation publique de l'enseignement - alors légitime. Les politiques éducatives de la III ${ }^{e}$ République, pourtant, ne se donnaient pas pour objectif de guider l'économie : des politiques de stabilisation de l'ordre économique et social allaient de pair avec une " école séparée de la production ${ }^{22} »$. Par quels mécanismes cette fonctionnalisation a-t-elle donc progressé au début des années 1950 ?

Cette fonctionnalisation résulte de dynamiques externes et internes à l'institution scolaire. Elle procède, premièrement, de l'investissement accru, de la part des milieux industriels et agricoles, de la formation comme facteur de production. L'analyse de leurs périodiques professionnels montre que les chefs d'entreprise et leurs fédérations multiplient des initiatives - parfois séparées, parfois communes, souvent concurrentes - qui, ensemble, développent la légitimité de l'enseignement dans les mondes du travail. Or, à la même époque, les réformateurs de certains segments de l'institution scolaire, en concurrence, tentent

\footnotetext{
17. Robert (A.), «La Quatrième République et les questions de l'égalité et de la justice dans l'enseignement du second degré : le changement sans la réforme ", Revue française de pédagogie, 159, 2007.

18. Mole (F.), "L'"égalité dans la diversité" : un modèle de justice à la préhistoire de l'école unique (18981914) », Revue française de pédagogie, 159, 2007.

19. Dorison (C.), « Orienter selon les aptitudes. Enjeux institutionnels et pédagogiques, 1936-1959 ", in D’Enfert (R.), Kahn (P.), dir., En attendant la réforme. Disciplines scolaires et politiques éducatives sous la IV République, Grenoble, Presses universitaires de Grenoble, 2010, p. 36-50.

20. Cf. respectivement Resnik (J.), "The Democratisation of the Education System in France after the Second World War: A Neo-Weberian Glocal Approach to Education Reforms ", British Journal of Educational Studies, 55 (2), 2007 et Prost (A.), L'enseignement s'est-il démocratisé ?, Paris, Presses universitaires de France, 1986.

21. Exposé des motifs du décret n 59-57 du 6 janvier 1959 portant réforme de l'enseignement public.

22. Lelièvre (C.), Nique (C.), La République n'éduquera plus. La fin du mythe Ferry, Paris, Plon, 1993.
} 
d' « ouvrir » l'enseignement aux acteurs extérieurs ; la fonctionnalisation procède alors, deuxièmement, de l'inscription, dans l'institution scolaire, des « besoins " de l'économie. Ce double mouvement de pédagogisation des mondes du travail et d'ouverture de l'enseignement les reformule, respectivement, en scolarisation des mondes du travail et d'économicisation de l'enseignement ; ensemble, ils contribuent à la fonctionnalisation de l'institution scolaire ${ }^{23}$.

\section{La pédagogisation des mondes du travail}

Les années 1944-1955 voient les instances représentatives de certains segments des milieux agricoles et industriels promouvoir l'enseignement avec une vigueur inédite. La formation est envisagée comme une ressource face à la brutale remise en cause de l'agriculture traditionnelle; elle donne également aux patrons de l'industrie, fragilisés depuis la Libération et inquiets du contexte de Guerre froide, l'espoir d'améliorer la productivité des salariés. Cet engouement ne se tourne toutefois pas vers les établissements publics d'enseignement : les dispositifs de transmission de savoirs et compétences censés résoudre ces problèmes économiques, « l'introduction du rapport pédagogique dans des fonctions et rôles qui en étaient exempts ${ }^{24}$ ", revêtent plutôt des formes non scolaires. Au début des années 1950, il n'est ainsi pas question de cette forme spécifique d'étatisation qu'est la scolarisation mais, seulement, d'une pédagogisation des mondes du travail.

\section{Former pour survivre}

À la Libération, « la formation véritable des travailleurs de l'agriculture n’a toujours pas commencé »; en 1955, 96,7 \% des exploitants agricoles n'ont reçu aucune formation technique ${ }^{25}$. L'intérêt pour la formation qui s'amorce alors marque ainsi une rupture historique.

Face aux brutaux changements techniques et économiques ${ }^{26}$, différents groupements ruraux, catholiques, familiaux ou agricoles appellent à développer l'enseignement pour la survie des campagnes. À l'avant-garde des mutations socio-professionnelles, le syndicalisme chrétien milite par et pour l'autoformation, qui permet de s'émanciper comme «personne » en même temps que de

\footnotetext{
23. Cet article, issu d'une recherche menée au sein du CURAPP (UMR CNRS-UPJV, Amiens), s'appuie sur l'analyse, principalement au cours des années 1946-1955, de revues généralistes agricoles, industrielles et scolaires, ainsi que sur des archives administratives de l'administration centrale de l'Éducation nationale et du Commissariat général au Plan. Cf. Bongrand (P.), La scolarisation des moeurs. Socio-histoire de deux politiques de scolarisation, en France, depuis la Libération, thèse de doctorat en science politique, Université de Picardie Jules Verne, 2009.

24. Beillerot (J.), La société pédagogique. Action pédagogique et contrôle social, Paris, Presses universitaires de France, 1982, p. 13.

25. Boulet (M.), Mabit (R.), De l'enseignement agricole au savoir vert, Paris, L'Harmattan, 1991, p. 22-26.

26. Gervais (M.), Jollivet (M.), Tavernier (Y.), La fin de la société paysanne. Histoire de la France rurale, de 1914 à nos jours, Paris, Seuil, 1976, vol. 4, p. 144-150.
} 
faire face aux mutations agricoles ${ }^{27}$. Pour les Cercles départementaux de jeunes, qui regroupent des agriculteurs de 15 à 30 ans, « le savoir est le pivot de l'agriculture de demain ${ }^{28} "$; les jeunes doivent en devenir des apôtres, c'est-à-dire « faire désirer la formation » en réaction à « l'insouciance des jeunes, la démission des familles et la carence des pouvoirs publics ${ }^{29} »$. Pour leur part, les Centres d'études techniques agricoles (CETA) réunissent des exploitants pour analyser collectivement leurs problèmes et élaborer des pratiques pédagogiques spécifiques («zones témoins", «foyers de progrès agricole ») ${ }^{30}$. De même certains responsables de mouvements familialistes catholiques - parfois issus de la Jeunesse agricole chrétienne (JAC) - promeuvent-ils une formation humaniste et salvatrice ${ }^{31}$. Le segment non confessionnel du mouvement de défense morale et matérielle des familles rurales crée, en 1945, un centre de formation professionnelle ménagère rurale ${ }^{32}$ et, en 1952, consacre son congrès national à la formation professionnelle. La Confédération nationale de la famille rurale (CNFR) nourrit également le mouvement en appelant les familles à éprouver ce besoin de services qu'elle peut précisément leur fournir ${ }^{33}$. Enfin, cette pédagogisation des campagnes est portée par des élites économiques. En 1951, la Chambre d'agriculture de la Somme crée un poste de conseiller agricole ${ }^{34}$ pour former ses adhérents à de nouvelles techniques de production ou de gestion. En collaboration ou en concurrence avec d'autres activités syndicales (les CETA) ou administratives (les directions des services agricoles), ces conseillers agricoles se multiplient ${ }^{35}$. L'instance qui réunit les Chambres d'agriculture (l'APPCA : Assemblée permanente des présidents des Chambres d'agriculture) tente de contenir l'essor des " jeunes $^{36}$ » en reprenant certaines de leurs revendications, parmi lesquelles la

27. Chosson (J.-F.), Les générations rurales, 1945-1990, Paris, LGDJ, 2003 ; Leprieur (F.), dir., JAC/MRJC, origines et mutations. Un mouvement de jeunesse au cour de la société française, Lyon, Chronique sociale, 1996 ; Purseigle (F.), Les sillons de l'engagement. Jeunes agriculteurs et action collective, Paris, L'HarmattanINJEP, 2004.

28. Citation de l'économiste Pierre Fromont en épigraphe du premier CNJ. Bulletin intérieur, 1, avril 1949. 29. «La formation professionnelle, problème numéro 1 de la jeunesse paysanne et de l'agriculture française ", CNJ. Bulletin intérieur, 16, novembre 1950.

30. Initié en région parisienne en 1945-1946, le mouvement devient en 1951 une Fédération qui compte alors vingt-cinq CETA. Houée (P.), Les étapes du développement rural. La Révolution contemporaine 19501970, Paris, Économie et Humanisme-Éditions ouvrières, vol. 2, 1972.

31. Par exemple : Colleu (P.), «En souvenir du splendide congrès de la JAC », L'Épi. Organe du mouvement d'action familial rural [d'Ille-et-Vilaine], juin 1954.

32. Pasquet (P.), "La formation des cadres, condition du développement de la formation ménagère rurale ", Chroniques paysannes. Service de presse bi-mensuel, 8, 1954.

33. Brun (F.), «Le XVe congrès national de la famille rurale », Directives rurales, 21, 1952.

34. Cf. Brives (H.), "La voie française : entre État et profession, l'institution du conseil agricole », in Rémy (J.), Brives (H.), Lémery (B.), dir., Conseiller en agriculture, Paris/Dijon, INRA/Educagri, 2006, p. 15-30 et Pinon (J.), «De la vulgarisation au développement », in Lebot (M.), Pesche (D.), dir., Campagnes en mouvement. Un siècle d'organisations paysannes en France, Paris, Charles Léopold Mayer, 1998, p. 79-93.

35. Ils passent de 1 à 83 entre 1951 et 1956. Caffarelli (J.-F. de), « Le Conseil agricole et la modernisation de l'agriculture française », Chambres d'agriculture, 637-638, 1978, p. 11.

36. Considérant que le syndicalisme agricole est structuré par l'opposition entre " jeunes » et " anciens », Yves Tavernier fait du rapport aux savoirs et à la formation l'un des éléments de ce clivage : « Les nouvelles 
formation, afin de devenir l'interlocuteur légitime des pouvoirs publics ${ }^{37}$. Soutenant la modernisation technique en contrepartie de la protection des prix, elle incite ses adhérents à se préoccuper de formation, publie des suppléments plus fréquents sur l'enseignement ${ }^{38}$ et enquête sur l'instruction des agriculteurs et l'offre de formation des Chambres départementales ${ }^{39}$. Soutenue par la Fédération nationale des syndicats d'exploitants agricoles (FNSEA) ${ }^{40}$, l'APPCA formalise des positions et rédige un contre-projet de loi sur la formation professionnelle. Elle défend la formation pour la survie des petites exploitations familiales, structures économiques fondamentales du monde agricole :

« Pour que nos entreprises agricoles demeurent d'abord, prospèrent ensuite, il faut, dans le respect des libertés de chacun, les rassurer sur l'avenir de leurs activités. Car le nombre des entreprises agricoles ne cesse de se comprimer ; mais ce mouvement se fait au hasard. Tel exploitant, mieux conseillé, aurait atteint un meilleur niveau de vie que celui qu'il trouvera dans son emploi urbain. Tel autre, dont l'exploitation n'est pas économiquement viable, se sacrifie en vain, alors qu'après une formation professionnelle accélérée, il pourrait trouver à gagner sa vie ailleurs... ${ }^{41}$ »

La modernisation est en marche : ceux qui l'ont compris se sont formés, ceux qui tardent doivent se former, ou disparaitre. La puissante Association générale des producteurs de blé, en pointe des associations de producteurs spécialisés, écoutée des pouvoirs publics ${ }^{42}$, développe dans cette perspective des "villages témoins » qui offrent une aide financière (semences, engrais, petit matériel) et technique ( mentor » qui sert de « conseiller quotidien ») afin de combler les «manques» :

" [Les] connaissances des agriculteurs auxquels on s'adresse, si on les compare à celles des élèves d'une école, sont parfois du niveau des premiers balbutiements de l'alphabet. Le seul moyen de les faire évoluer est, après avoir acquis leur confiance, de les prendre par la main et de les suivre pas à pas dans leurs

\footnotetext{
élites paysannes, formées le plus souvent par les mouvements d'action catholique, [...] cherchent à s'instruire, parlent "revenu", rémunération, capital d'exploitation, organisation du travail. Elles refusent l'état héréditaire d'agriculteurs. La volonté des jeunes de s'intégrer dans le monde moderne, d'acquérir une grande compétence technique pour améliorer les méthodes de production, de connaître les mécanismes de l'économie afin de n'être plus dominés par le marché, les conduit à mettre en cause l'individualisme traditionnel de leurs parents.» Tavernier (Y.), « Le syndicalisme paysan et la politique agricole du gouvernement. Juin 1958 - avril 1962 ", Revue française de science politique, 12 (3), 1962, p. 601.

37. En vertu d'un protocole signé le 8 février 1951 avec l'UGA et la FNSEA, l'APPCA représente les intérêts paysans en matière de formation professionnelle.

38. Suivant un rythme irrégulier mais croissant, un supplément « enseignement agriculture » est annexé à partir de septembre 1952 à la revue de l'APPCA.

39. Chambres d'agriculture, 24 (26), 1953, supplément.

40. Chambres d'agriculture, 24 (41), 1953.

41. Blondelle (R.), La politique agricole de demain, supplément à Chambres d'agriculture, 15 décembre 1955, p. 29-30.

42. Pesche (D.), Le syndicalisme agricole spécialisé en France. Entre la spécificité des intérêts et le besoin d'alliances, Paris, L'Harmattan, 2000.
} 
travaux. À quiconque trouvera que ce tableau est noirci, il n'est pas difficile d'apporter la preuve du contraire ${ }^{43}$. »

Cette posture vulgarisatrice, soucieuse de diffuser des techniques constituées ailleurs, s'oppose certes à l'approche des jeunes catholiques, pour qui le savoir est produit par les acteurs de terrain dans une logique émancipatrice. Publiques comme privées, ces différentes parties au mouvement de pédagogisation concourent cependant à une surenchère, indissociablement discursive et pratique, de formation. Elles y contribuent d'autant plus intensivement que leur régulation est à l'agenda politique. Si, par exemple, le MFR consacre ses journées sociales en 1950-1951 à l'apprentissage et à l'orientation professionnelle, c'est pour avertir qu' « une nette poussée du ministère de l'Éducation nationale (dont on connaît la tendance) risque de mettre [les familles rurales] dans une situation de fait difficile à améliorer par la suite ${ }^{44} »$. Instances professionnelles, partis politiques et ministères élaborent des projets de statut de la formation professionnelle ${ }^{45}$ : la chronique de leurs rivalités entretient l'injonction à former et les débats, concentrés sur les modalités, illustrent le consensus en faveur de la nécessité de former ${ }^{46}$.

\section{Former pour " manager 》}

Si la pédagogisation affecte également les milieux industriels, le point de départ, en matière de formation professionnelle, n'est pas le même. Une originalité du moment tient à ne plus concentrer la formation professionnelle en amont du recrutement, mais à en faire un instrument de gestion des personnels tout au long de leur carrière. Les premiers responsables des services du personnel dans les grandes entreprises ${ }^{47}$ contribuent à ce qui constitue l'une des innovations les plus sensibles des politiques de personnel au cours du XXe siècle ${ }^{48}$.

Plusieurs facteurs conduisent à considérer que la formation peut payer ${ }^{49}$. L'évolution « objective » du travail, tout d'abord, crée de nouvelles fonctions

43. Mennesson (H.), David (É.), « Les villages témoins », Terres de France et de l’Union française, 7, 1954, p. 51.

44. «Les journées sociales rurales 1950-1951 », Directives rurales, 40, 1950, p. 6-7. Cette rivalité s'illustre dans la création de cours agricoles publics dans des territoires déjà dotés en maisons familiales, au détriment d'espaces encore dénués d'équipement, cf. Lelorrain (A.-M.), « Le rôle de l'école laïque et des instituteurs dans la formation agricole, 1870-1970», Histoire de l'éducation, 65, 1995, p. 59-60.

45. Lelorrain (A.-M.), Bobbio (M.), dir., L'enseignement agricole et vétérinaire de la Libération à nos jours. Textes officiels avec introduction, notes et annexes, Dijon-Paris, Éducagri-INRP, p. 24-29 et Leveau (R.), "L'enseignement et la vulgarisation agricoles ", in Fauvet (J.), Mendras (H.), dir., Les paysans et la politique, Paris, Armand Colin, 1958, p. 269-280.

46. Cf. Châtelain (R.), «Les résultats d'une grande enquête sur l'enseignement agricole », Terres de France et de l'Union française, 10, 1954.

47. Tanguy (L.), «Les promoteurs de la formation en entreprise (1945-1971) », Travail et emploi, 86, 2001. 48. Fombonne (J.), Personnel et DRH. L'affirmation de la fonction personnel dans les entreprises. France (18301990), Paris, Vuibert, 2001, p. 429.

49. Suivant la formulation incitative d'un rapport de mission de productivité : «Actuellement, pour ellemême, l'industrie emploie de grands moyens, car elle estime que la formation paye ». Cadres et maîtrise. Leur formation en tant que chefs aux États-Unis, Paris, SADEP, 1952, p. 21. 
qui appellent formation. Or certaines des branches concernées par ces évolutions occupent une position éminente dans les institutions représentatives du patronat. C'est le cas de l'Union des industries et des métiers de la métallurgie (UIMM), par laquelle le Conseil national du patronat français (CNPF) enregistre les premières pratiques de formation initiées par des branches, les met en avant à titre exemplaire et, ce faisant, appelle les chefs d'entreprise à les généraliser comme politiques de formation. Les acteurs et théories de l'organisation du travail, contribuent également au mouvement ; influencés et portés par la campagne de productivité ${ }^{50}$, ils font parfois de la formation une compétence professionnelle et, reconsidérant les missions de certains postes de travail (qui appellent mise à jour, donc formation), en élèvent le niveau (donc suggèrent une formation initiale plus longue). Enfin, depuis la Libération, le volontarisme de l'État en matière de formation professionnelle (via l'enseignement technique et la formation professionnelle accélérée) attire les patrons sur un terrain où combattre le « dirigisme ». Face à un champ qui leur échappe en même temps qu’il se constitue, des entreprises ou groupements représentatifs entrent ainsi dans la course : pour ne pas laisser le terrain libre à la scolarisation, ils s'engagent dans le mouvement de pédagogisation, travaillant à donner écho et réalité à ce qui deviendra un mot d'ordre de l'UIMM : « La formation du personnel d'encadrement est au premier plan des préoccupations des chefs d'entreprise ${ }^{51}$.

L'un des ressorts de ce développement de la formation en entreprise réside dans son appréhension comme instrument de gouvernement des salariés ${ }^{52}$. Marqués par des grèves massives et le refroidissement des relations internationales, les milieux patronaux se préoccupent en effet de la collaboration de leurs personnels. Le problème s'impose notamment au Centre national de l'organisation française $(\mathrm{CNOF})^{53}$ qui, lors de ses journées annuelles de 1948 et 1949, organisées par Rolf Nordling ${ }^{54}$, s'interroge sur les moyens de maitriser les « facteurs de solidarité humaine dans l'entreprise » et se met à " la recherche des facteurs de la paix sociale ». La formation apparaît alors comme une solution : associée à la promotion, elle promet à tout salarié qu'il peut accéder aux positions hiérarchiquement supérieures et, par là, renforce le " climat » au sein

\footnotetext{
50. Boulat (R.), Jean Fourastié, la productivité et la modernisation de la France (années trente - années cinquante), thèse pour le doctorat d'histoire, Université de Franche-Comté, 2006.

51. UIMM, Formation. 8 réalisations, s.l.n.d. [1956 d'après la BnF, 8 - R - 59151 (1)].

52. Brucy (G.), Caillaud (P.), Quenson (E.), Tanguy (L.), Former pour réformer. Retour sur la formation permanente (1945-2004), Paris, La Découverte, 2007.

53. Le CNOF, créé en 1926, entend regrouper les professions et doctrines d'organisation scientifique du travail actives en France. Entre 1946 et 1952, il compte environ trois mille membres.

54. Suite aux grèves de 1936, R. Nordling organise, dans le cadre du groupe réuni autour d'A. Detœuf, des rencontres franco-suédoises où l'entente entre syndicats ouvriers et patronaux frappe les esprits. Il est l'auteur d'articles aux titres éloquents : "Nouvelle étape vers la fusion des classes en Suède ", Nouveaux Cahiers, 46, 1939 ; " Genèse de l'harmonie sociale en Suède ", Centre des jeunes patrons, 18, 1946 ; "L'enseignement de l'organisation en Suède et ses conséquences sociales ", CNOF. Revue mensuelle de l'organisation, 20 (3), 1946.
} 
d'une entreprise. Cette approche se projette notamment dans un programme qui permet, entre autres, d'enseigner aux contremaittres comment encadrer des ouvriers : le TWI (Training Within Industry) ${ }^{55}$. En 1948, R. Nordling réunit les principales instances de l'organisation scientifique du travail pour importer ce programme américain ${ }^{56}$. La revue du CNOF lui accorde une place croissante et retranscrit l'enthousiasme de R. Nordling, d'après qui « l'extension rapide » du TWI, œuvre d'un « nouveau Christophe Colomb », « devrait donner en France des résultats sensationnels pour l'économie et, dans une certaine mesure, pour la paix sociale du pays ${ }^{57} »$. La campagne de productivité relaie également ce discours, ses outils et ses exemples : le rapport sur les «méthodes de formation et de perfectionnement des cadres supérieurs et subalternes (maitrise) de l'industrie américaine » confirme que les programmes TWI, « certainement à la base de toute l'évolution de l'Amérique en matière de formation des cadres, ont apporté à l'Économie américaine moderne un de ses plus puissants outils de production. [...] L'expérience prouve que l'application de cet enseignement crée un climat de compréhension, apporte la paix sociale et favorise la production ${ }^{58}$. » Sans être incarnée par le TWI, la même perspective se retrouve, enfin, au CNPF. En 1952, son président Georges Villiers, issu de l'UIMM ${ }^{59}$, présente la formation comme levier de développement économique et suggère qu'à tout problème de productivité, formation est bonne ${ }^{60}$. Il argumente en y voyant une manière de convaincre les salariés que l'ascension interne est possible :

«Et je crois que nous devons nous pencher sur le problème de la promotion depuis la base, depuis les ouvriers, pour utiliser au mieux toutes les valeurs de ce pays. Beaucoup a déjà été fait dans ce sens, mais il faut que nous fassions véritablement en sorte que nul, quelle que soit son origine, n'ait le droit de dire qu'il n'a pas eu la possibilité de s'élever dans l'industrie ou le commerce au niveau que justifiaient ses qualités et ses efforts. Si ce qui existe n'était pas suffisant, je voudrais que vous soyez d'accord pour reconnaître qu'il faut aller plus loin dans cette voie. Nous devons aussi nous pencher sur le problème de la promotion au sein même de l'entreprise ou de la profession. Cette promotion doit

\footnotetext{
55. Mis au point au cours de la Seconde Guerre mondiale par l'administration fédérale américaine éponyme, le TWI est à l'origine un instrument qui met la formation au service de l'économie ( "More Production Through Training »), en même temps qu'il met la science au service de la guerre (le programme s'inspirant des travaux de psychologie sociale menés au cours des années 1930 à l'usine Hawthorne par l'équipe d'Elton Mayo). Cf. Breen (W. J.), « Social Science and State Policy in World War II: Human Relations, Pedagogy, and Industrial Training, 1940-1945 », The Business History Review, 76, 2002.

56. Cf. Jean Fombonne, Personnel..., op. cit., p. 446-458.

57. CNOF. Revue mensuelle de l'organisation, septembre 1950, p. 94.

58. Cadres et maîtrise..., op. cit., p. 20, 64 et 65 . Cette mission était présidée par R. Nordling.

59. Dirigeant d'un syndicat de la métallurgie à Lyon avant-guerre, G. Villiers avait déjà développé la formation, cf. Villiers (G.), Témoignages, Paris, France-Empire, 1977, p. 15-16 ; Fraboulet (D.), « Georges Villiers (1899-1982) ", in Daumas (J.-C.), dir., Dictionnaire historique des patrons français, Paris, Flammarion, 2010, p. 693-695.

60. Bulletin du CNPF, 78, 1952.
} 
être rendue possible partout grâce au développement systématique des moyens qui permettent aux cadres de compléter sans cesse leur formation ${ }^{61}$. »

Cet extrait illustre la congruence des discours de politiques d'entreprise avec ceux qui accompagneront les réformes de démocratisation scolaire, où enseignement, justice, mérite et promotion sociale font système. Au sujet des entreprises, il montre non seulement que la formation s'adosse bien à des relations de pouvoir mais, également, qu'elle est une manière spécifique de les légitimer. Associer la formation à la pacification des relations de travail, c'est en effet faire des différents étages de la ligne hiérarchique, vus du bas, des positions souhaitables et accessibles par la formation et, vus du haut, des positions de pouvoir acquises de manière juste et rationnelle. Si à l'échelle d'un individu, la formation signifie espoir d'ascension, à l'échelle de l'organisation, la formation renvoie à un organigramme pyramidal, dont les étages se multiplient. Le contexte exhorte précisément à généraliser cette forme pédagogisée des relations de pouvoir dans l'entreprise : d'après des préceptes forts de la campagne de productivité, les agents de tous les échelons intermédiaires de la ligne hiérarchique peuvent être envisagés comme des chefs, et tous peuvent être formés à l'autorité : l'intérêt pour la formation est l'une des faces de la révision du rôle et des tâches de ces catégories intermédiaires ${ }^{62}$. L'essor de ces discours associant formation, position et autorité concourt à la scolarisation des mœurs, où des performances dans l'institution scolaire peuvent légitimer des positions de pouvoir dans l'univers professionnel.

\section{Former plutôt que scolariser}

Un trait majeur de cet engouement pour la formation, au début des années 1950, tient à ce qu'il n'est pas projeté vers l'institution scolaire. Dans le monde rural, les professionnels de l'agriculture entreprennent de former eux-mêmes les populations. Dénonçant le sous-développement de l'enseignement public, ils insistent sur l'incapacité de l'État à répondre à des besoins que les populations ont commencé d'identifier et résoudre seules. L'État doit certes rattraper son retard, mais sans se substituer à ceux qui se sont lancés les premiers : " [Il] doit jouer son rôle, tout son rôle, mais rien que son rôle ${ }^{63}$. " L'étatisation est acceptée sous la forme d'un accroissement de l'offre et du financement publics, non comme monopole :

«Sans contester à l'État son droit de contrôle et de coordination, on peut estimer indésirable qu'il impose aux familles paysannes, par voie d'autorité, un système post-scolaire auquel les organismes ruraux seraient étrangers. L'emprise grandissante de la Puissance publique sur la jeunesse inquiète tous ceux

61. Journée interprofessionnelle de la productivité à Marseille, 8 mai 1952, BnF, 4 - R PIECE - 4649.

62. Boltanski (L.), Les cadres. La formation d'un groupe social, Paris, Minuit, 1982.

63. Nové-Josserand (F.), "La formation professionnelle agricole privée et ménagère privée ", Terres de France et de l'Union française, 10, 1954, p. 32. 
qui pensent que l'éducation des enfants est d'abord l'affaire de la famille et non pas exclusivement celle des mandataires d'une Technocratie souveraine. Cette inquiétude est particulièrement ressentie dans le monde rural. Elle constitue l'un des aspects du malaise dont souffre la paysannerie française. Ayant le goût de l'indépendance et des libertés, celle-ci est prête à assumer toutes ses obligations en ce qui concerne la formation professionnelle et ménagère de ses enfants. Ce qu'elle demande à l'État, c'est qu'il l'aide à accomplir sa tâche et non qu'il [se] substitue purement et simplement à elle ${ }^{64}$.»

Cette attitude se retrouve dans l'industrie. Dès sa première assemblée générale, le CNPF adopte une motion « contre l'envahissement de l'État dans le domaine de l'apprentissage ${ }^{65} »$. Sa sous-commission compétente milite pour « l'organisation de la formation professionnelle et de l'apprentissage dans le cadre de la profession ${ }^{66}$ » et s'oppose à l'emprise croissante des fonctionnaires sur les programmes, sujets et jurys d'examen ${ }^{67}$. Montrant l'exemple en mettant en place une formation pour les patrons ${ }^{68}$, le CNPF encourage les entreprises à pourvoir elles-mêmes à leurs besoins :

«Car il ne faut pas se dissimuler que les écoles publiques et même les écoles créées par les associations ne pourront jamais entièrement satisfaire les besoins si variés et si variables en techniciens. Les organisations syndicales devront les compléter, en créant elles-mêmes des écoles satisfaisantes, les prolonger par des stages et surtout fortifier la formation de leurs techniciens et cadres actuels et futurs par des cours ou sessions. Plusieurs industries en ont déjà donné l'exemple ${ }^{69}$.»

Contre l'étatisation, cette pédagogisation se veut aussi distincte d'une scolarisation. Dans le monde rural, les tenants d'une formation professionnelle adaptée aux temporalités et préoccupations locales, orientée par les politiques ou professions agricoles, s'opposent aux défenseurs d'une formation générale, à plein-temps, relativement détachée de la conjoncture agricole (voire rurale) ${ }^{70}$. Si le processus conduit la majorité du monde agricole à accepter une intervention publique, c'est à condition qu'elle ne soit pas scolaire ${ }^{71}$. Dans le monde de

64. Pasquet (P.), « La formation des jeunes ruraux est d'abord l'affaire des familles », Documentation familiale et rurale. Service de presse bi-mensuel de la CNFR, 19, 1953, p. 2.

65. Bulletin du CNPF, 3, 1947, p. 10.

66. Bulletin du CNPF, 1, 1946, p. 7.

67. Par exemple Bulletin du CNPF, 11, 1947-1948, p. 11-12 et 16, 1948, p. 16, ainsi que Brucy (G.), Histoire des diplômes de l'enseignement technique. 1880-1965. L'État, l'école et la certification des compétences, Paris, Belin, 1998, p. 153-159.

68. Bulletin du CNPF, 78, 1952, p. 2-4.

69. Bulletin du CNPF, 129, 1955, p. 93.

70. Cette opposition fait écho à la rivalité traditionnelle entre administrations de l'Agriculture (soutenue par ses ingénieurs agricoles et les représentants professionnels) et de l'Éducation nationale (soutenue par ses instituteurs et institutrices ainsi que par les communistes), cf. Cépède (M.), Weill (G.), L'agriculture, Paris, Presses universitaires de France, 1965.

71. «Ce que les agriculteurs pensent de l'école primaire. Une enquête des Chambres d'agriculture », Fiches rurales, 109, 1955, p. 31-34. 
l'industrie, la forme scolaire d'enseignement est jugée inadaptée, trop concentrée sur la diffusion de savoirs formels au détriment de la production de comportements et compétences ${ }^{72}$. Significativement, l'enseignement scolaire est disqualifié pour son incapacité à légitimer le pouvoir d'un chef : à la question « Les contremaîtres ne peuvent-ils être gênés si les ouvriers qu'ils dirigent savent qu'ils vont à l'école ? Cela ne crée-t-il pas chez eux une sorte de complexe? », un formateur répond ainsi : «Un effort doit être fait de la part des organisateurs pour éviter tout ce qui peut rappeler l'école : les réunions ne doivent surtout pas être appelées des cours ${ }^{73}{ }^{\prime}$. Les techniques du TWI sont, une fois encore, paradigmatiques : elles n'isolent pas de lieu spécifique de formation, elles recherchent la convivialité, l'anthropologie spécifique qu'elles visent méthodiquement associe aspirations individuelles, épanouissement dans la carrière (plus que dans le travail) et idéal de bonnes « relations humaines ». L'ensemble esquisse un mode d'éducation irréductible à la forme scolaire de socialisation ${ }^{74}$.

Agricole ou industrielle, enjeu de rivalités institutionnelles, la formation est donc développée comme outil d'émancipation matérielle ou spirituelle, facteur de compétitivité ou encore instrument de maintien de l'ordre au sein des usines. Cette diversité décline un mot d'ordre commun : que ce soit plus, mieux ou bien autrement, il faut former. Il reste cependant à déterminer la division du travail de formation entre acteurs publics et privés. Dans la mesure où l'engouement pour la formation est fortement opposé à l'État, ainsi qu'à la forme scolaire d'enseignement, la réglementation de cette effervescence pédagogique est décisive pour la tournure du processus. Cette pédagogisation des mondes du travail aurait en effet pu jouer comme obstacle - plutôt que comme facteur propice - à l'avènement d'un système scolaire articulé aux « attentes » économiques. Quels sont donc les éléments qui ont contribué à nouer cette évolution à celle de l'institution scolaire? Comment la pédagogisation des mondes du travail s'est-elle spécifiée en étatisation et, plus précisément, en scolarisation?

\section{La fonctionnalisation de l'institution scolaire}

Dans ce contexte, l'évolution de l'institution scolaire se comprend au regard de dynamiques internes. À la Libération, l'enseignement est organisé en quatre séries d'établissements perçus comme parallèles, étanches et concurrents. Ces quatre ordres d'enseignement (respectivement technique, du premier degré, du second degré, supérieur) sont dotés de diplômes, personnels, méthodes, programmes, administrations, publics et débouchés professionnels propres. Leurs directions administratives, profitant de l'instabilité gouvernementale, mènent séparément la réforme de leur segment spécifique. Dans leur rivalité, « l'ouverture à la vie »

72. Cf. Bulletin du CNPF, 2, 1946, p. 7, ou 84, 1954, p. 53.

73. "Une expérience de formation à la SNECMA », Cégos, 1955, document PS 130, p. 11.

74. Vincent (G.), L'école primaire française. Étude sociologique, Lyon, Presses universitaires de Lyon, 1980. 
de l'enseignement peut se présenter comme une stratégie où les «besoins de l'économie » apparaissent comme une ressource.

\section{L'ouverture de l'enseignement}

Établir une offre d'enseignement à visée professionnelle n'est pas une nouveauté des années 1950 : tout ou partie des établissements d'enseignement technique ou supérieur assument déjà cette vocation. Néanmoins, la logique économique de préparation au travail progresse sensiblement dans certains segments de l'enseignement général, où des dynamiques institutionnelles de développement ou de concurrence conduisent à endosser de nouvelles « demandes sociales $»$ de formation ${ }^{75}$.

Tel est le cas, d'une part, de l'enseignement du second degré. Depuis la Libération, son organisation est remise en cause pour des raisons politiques (iniquité de son recrutement), logistiques (pénurie d'enseignants), pédagogiques (archaïsme des programmes, échecs au baccalauréat) et institutionnelles (rivalité, entre autres, avec l'enseignement du premier degré). Jusqu'en juillet 1951, le directeur de cet ordre d'enseignement est Gustave Monod, engagé de longue date, comme enseignant puis administrateur, dans les mouvements de l'éducation nouvelle ${ }^{76}$. Il compte sur la modernisation des programmes et pédagogies de l'enseignement secondaire, dont « l'ouverture » inclurait une formation manuelle et technique, et, pour les élèves susceptibles d'échouer, sur le développement de l'enseignement technique. Son successeur Charles Brunold est en revanche plus conservateur. Instituteur qui a gravi, y compris pendant le régime de Vichy, les différents échelons de la hiérarchie de l'Éducation nationale ${ }^{77}$, il souhaite préserver les traits typiquement secondaires de cette filière : son projet consiste à lui adjoindre un nouvel enseignement du second degré court, qui ne donnerait pas accès au baccalauréat et délesterait ainsi la filière traditionnelle sans nourrir pour autant les filières de scolarisation post-obligatoires administrées par l'enseignement du premier degré ${ }^{78}$. Or si ce projet, qui attise les rivalités entre syndicats enseignants du primaire et du secondaire ${ }^{79}$, échoue en juin 1953 au sein de l'Éducation nationale, il est relayé sur une scène alternative. La commission de la main-d'œuvre pour le II ${ }^{e}$ Plan voit en effet dans l'enseignement court du second degré une solution au besoin, qu'elle diagnostique alors, en personnels de niveau d'études intermédiaire - les cadres et techniciens.

\footnotetext{
75. Briand (J.-P.), Chapoulie (J.-M.), « L'institution scolaire et la scolarisation : une perspective d'ensemble ", Revue française de sociologie, 34, 1993.

76. Chapoulie (J.-M.), « Entre le lycée d'élite et le lycée de masse. Paul Langevin, Gustave Monod et les réformes de l'enseignement secondaire de 1936-1939 et de 1944-1951 », in Caspard (P.), Luc (J.-N.), Savoie (P.), dir., Lycées, lycéens, lycéennes. Deux siècles d'histoire, Lyon, INRP, 2005, p. 145-157.

77. Ferrez (J.), Au service de la démocratisation. Souvenirs du ministère de l'Éducation nationale. 1943-1983, Lyon, INRP, 2004, p. 51.

78. L'Éducation nationale, 14 et 15, 1953 ; Robert (A.), « La Quatrième République... », art. cit.

79. Cf. Brucy (G.), Histoire de la FEN, Paris, Belin, 2003, p. 259-281.
} 
Dans le rapport final de la commission, la réforme de l'enseignement se trouve alors justifiée dans une nouvelle perspective : les problèmes d'engorgement du second degré n'y sont plus envisagés en termes logistiques ou politiques, mais sous l'angle de leur irrationalité économique ${ }^{80}$. Déplorant, par exemple, l'excès d'étudiants juristes et littéraires, la commission ne suggère pas de leur créer des débouchés, mais de former et d'orienter ces étudiants différemment, de manière plus ajustée aux impératifs économiques (en l'espèce : en créant des diplômes de technicien). Pour nouer ainsi les fils de la réforme de l'enseignement avec ceux des politiques d'emploi, le rapport identifie une variable d'ajustement non dans l'emploi, mais dans l'enseignement, dont la réforme s'apparente alors à une économicisation.

L'enseignement supérieur connaît un mécanisme analogue. L'enseignement du second degré pèse en effet sur son recrutement (puisqu'il en conçoit le titre d'accès : le baccalauréat), en soustrait les meilleurs élèves (orientés vers les grandes écoles) et impose aux activités pédagogiques les méthodes et programmes des concours d'agrégation (auxquels l'enseignement supérieur prépare les étudiants $)^{81}$. Dans la lignée de la commission Langevin, qui veut « bien séparer » les enseignements du second degré et supérieur ${ }^{82}$, les directeurs successifs de l'enseignement supérieur entendent simultanément donner une place aux disciplines scientifiques récentes, sans agrégation, et former à de nouvelles professions qualifiées. Les physiciens Pierre Auger (1945-1948) et Pierre Donzelot (19481953) créent ainsi les écoles nationales supérieures d'ingénieurs et encouragent l'académisation des sciences de l'ingénieur ${ }^{83}$. Directeur de l'enseignement supérieur à partir de 1953, Gaston Berger, philosophe venu à la psychologie, mais également ancien chef d'entreprise qui a construit sa carrière à l'interface entre milieux économiques et scientifiques ${ }^{84}$, agit de même en faveur des sciences humaines ${ }^{85}$. Il les défend notamment au nom d'une utilité pour l'industrie, terrain d'application pertinent puisque le management apparaît alors comme un métier nouveau et nécessaire auquel il est possible de former. Sous la direction de G. Berger sont ainsi créés des instituts de sciences appliquées. En mai 1955, en coopération avec le Commissariat général à la productivité, ses

80. "Rapport général de la commission de la main-d'œuvre du Commissariat général au Plan », Revue française du travail, 9 (3), 1954, p. 107 et 108. Cf. Charlot (B.), Figeat (M.), Histoire de la formation des ouvriers. 1789-1984, Paris, Minerve, 1985.

81. Cette critique est portée par Lucien Febvre, membre de la Commission Langevin-Wallon, par exemple dans : « Plaidoirie pour une révolution », L'Éducation nationale, 6, 1947.

82. Commission d'étude pour la réforme de l'enseignement, "Procès-verbal sommaire » de la $42^{\mathrm{e}}$ séance, 23 mars 1946, p. 2, archives Henri Wallon, Centre historique des Archives nationales (AN) 360 AP 4.

83. Grossetti (M.), Detrez (C.), « Sciences d'ingénieurs et sciences pour l'ingénieur. L’exemple du génie chimique », Sciences de la société, 49, 2000.

84. Guiader (V.), Socio-histoire de la prospective. La transformation d'une entreprise réformatrice en expertise d'État, thèse pour le doctorat de science politique, Université Paris Dauphine, 2008.

85. Cf. les premiers numéros de la Revue de l'enseignement supérieur significativement consacrés à «L'université dans la vie sociale et économique », 4, 1956, et à «L’université et le progrès technique », 1 et 2, 1957. 
services élaborent un programme de formation universitaire à l'administration des entreprises ${ }^{86}$. Douze universités sont alors habilitées à délivrer un nouveau certificat, dont les publics, corps enseignants, intitulés de cours et formes pédagogiques rapprochent l'enseignement supérieur des milieux économiques. Ces instituts d'administration des entreprises incorporent et intègrent à l'université le mouvement de pédagogisation des mœurs professionnelles envisagé plus haut, suivant le résumé qu'en donne un acteur :

"Il existe en France d'excellentes institutions qui préparent les étudiants à la vie des affaires. Sauf exception, elles ne sont pas, et ceci est presque unique au monde, rattachées aux universités. Il a semblé, au cours des dernières années, qu'il fallait mettre en place des organismes nouveaux, non pour concurrencer les institutions existantes, mais pour répondre à des besoins nouveaux et accrus. Les "relations humaines" au sens large, les problèmes de gestion et de contrôle, les structures et la hiérarchie des entreprises se transforment et deviennent plus complexes. En outre, une tendance s'est développée qui cherche à renforcer les liens entre universités et industries. Cette tendance a trouvé d'ardents défenseurs aussi bien dans les milieux industriels que dans les milieux universitaires. Ces raisons sont directement ou indirectement à l'origine de la création du certificat d'aptitude à l'administration des entreprises. Il a semblé, en particulier, nécessaire de permettre aux étudiants pourvus d'une formation d'enseignement supérieur d'être plus rapidement et mieux utilisés par les entreprises dont ils connaissaient insuffisamment les structures et les problèmes ${ }^{87}$. "

Comme cet extrait l'indique, en matière de réforme, le rapprochement entre enseignement et économie repose sur de nombreuses assertions relatives à l'évolution du travail comme de l'université. Des savoirs et producteurs de savoirs précis y autorisent.

\section{Les termes d'un rapprochement entre enseignement et économie}

La formulation d'un enseignement fonctionnel pour l'économie est solidaire de savoirs qui documentent, d'une part, les besoins de l'économie en flux de diplômés et, d'autre part, les effets de l'enseignement sur l'économie. Dans des revues, commissions d'experts ou lieux de formation d'administrateurs, ces savoirs sont diffusés par des enseignants-chercheurs qui militent pour le développement de savoirs ou institutions scientifiques, et exportent leur croyance dans la puissance de la formation et du savoir. En matière agricole, la nécessité de former aux techniques de production ou de gestion est ainsi démontrée par l'agronome René Dumont, professeur à l'Institut national agronomique

86. Revue de l'enseignement supérieur, 1, 1956, p. 64. Nioche (J.-P.), Kipping (M.), « Politique de productivité et formation à la gestion (1945-1960) : un essai non transformé », Entreprises et histoire, 14, 1997. Chessel (M.-E.), Pavis (F.), Le technocrate, le patron et le professeur. Une histoire de l'enseignement supérieur de gestion, Paris, Belin, 2001, p. 32.

87. Goetz-Girey (R.), "Une création récente : le certificat d'aptitude à l'administration des entreprises », Revue de l'enseignement supérieur, 4, 1956, p. 81-82. 
de Paris, actif au Plan et collaborateur du CETA ${ }^{88}$, et les économistes ruraux Pierre Fromont (Faculté de Droit de Paris) et Jean Chombart de Lauwe (Institut national agronomique de Grignon). Le démographe Alfred Sauvy et l'économiste Jean Fourastié, également engagés dans l'institutionnalisation de savoirs utiles aux politiques publiques ${ }^{89}$, défendent la pertinence scientifique et l'utilité politique d'hypothèses relatives à l'évolution de la structure de la population active et, partant, des besoins en catégories de diplômés. En 1952-1954, ils réorientent en ce sens la commission de la main-d'œuvre du Plan. Contrairement aux années précédentes, cette commission planifie en effet sur la base de prévisions économiques plutôt que par le recueil des attentes (jugées malthusiennes) des partenaires sociaux, elle insiste sur les besoins futurs en professions qualifiées (cadres, scientifiques, techniciens) et, surtout, elle préfere modeler la main-d'œuvre plutôt que de la considérer comme une donnée appelant une action publique en matière de placement professionnel ou de reconversion. A. Sauvy et J. Fourastié s'ils divergent sur la teneur du changement à venir ${ }^{90}$, insistent sur les conséquences à en tirer en matière scolaire : dans cette perspective, ils élargissent les travaux de la commission de la main-d'œuvre au secteur de l'enseignement ${ }^{91}$. Ce coup de force s'explique moins comme la mobilisation de savoirs déjà constitués que comme le succès de savants engagés pour institutionnaliser leurs hypothèses. Il relaie la pédagogisation des mondes professionnels et amorce le mouvement qui débouchera, au milieu des années 1960, sur la mise en équivalence de la formation avec l'emploi dans des nomenclatures administratives ${ }^{92}$. En 1952-1954, la formation est pourtant ignorée des théories contemporaines de la productivité et l'économie de l'éducation n'est pas encore constituée comme discipline ${ }^{93}$; l'enseignement se profile cependant déjà comme élément d'une politique économique.

Réciproquement, des savoirs portent sur les enjeux économiques de l'enseignement. En différents segments de l'institution scolaire circulent des discours qui envisagent les élèves d'après leur origine ou leur avenir socio-professionnels. Il en va ainsi des approches en termes de démocratisation de l'enseignement ${ }^{94}$ : à chacune des rentrées 1953, 1954 et 1955, la revue de l'INED, Population,

\footnotetext{
88. Houssel (J.-P.), « Des débuts de la révolution fourragère dans le lyonnais à la modernisation en petite culture », Géocarrefour, 81 (4), 2006.

89. Rosental (P.-A.), L'intelligence démographique. Sciences et politiques des populations en France (19301960), Paris, Odile Jacob, 2003.

90. Chapoulie (J.-M.), "Les nouveaux spécialistes des sciences sociales comme "experts" de la politique scolaire en France 1945-1962 ", Genèses, 64, 2006.

91. Cf. les archives de la commission, AN 80 AJ 49 et 85.

92. Tanguy (L.), «La mise en équivalence de la formation avec l'emploi dans les IV et Ve plans (1962-1970) », Revue française de sociologie, 43 (4), 2002.

93. Segré (M.), Tanguy (L.), Lortic (M.-F.), « A New Ideology of Education », Social Forces, 50, 1972 ; Vinokur (A.), « De la scolarisation de masse à la formation tout au long de la vie. Essai sur les enjeux économiques des doctrines éducatives des organisations internationales ", Éducation et sociétés, 12, 2003. 94. Ravon (B.), L’«échec scolaire». Histoire d'un problème public, Paris, In Press, 2000.
} 
démontre que des élèves " aptes » sont perdus pour le système scolaire en raison de leur milieu social ; les services d'orientation professionnelle enquêtent également en 1955 sur la proportion d'élèves qui ne se présentent pas à l'entrée en sixième alors que leurs aptitudes sont au moins équivalentes à celles des élèves scolarisés dans le premier cycle du second degré ${ }^{95}$. Il en va également ainsi d'approches qui, en termes de psychologisation ou d'individualisation de l'enseignement, envisagent les enfants en tant qu'ils sont prédisposés à des carrières scolaires et professionnelles différentes. Le directeur de la section des affaires générales du cabinet du ministre Jean Berthoin, André Le Gall, tout comme le directeur de l'enseignement supérieur, Gaston Berger, sont des auteurs et promoteurs centraux de la caractérologie, branche de la psychologie scientifique qui postule l'existence de structures congénitales constitutives du « caractère », lequel peut être mis en correspondance avec des familles de métier. A. Le Gall écrit ou écrira de nombreux ouvrages plaidant pour structures ou pédagogies soucieuses d'identifier et d'accompagner les différentes " personnalités ». Ces positions s'apparentent ainsi à celles du décret de 1959, qui énonce que de « jeunes esprits [...] trouveraient mieux leur voie dans l'enseignement technique à l'un ou à l'autre de ses niveaux ", par opposition à " des intelligences auxquelles les enseignements longs, technique ou secondaire, vaudraient leur accomplissement véritable».

S'ils permettent de lier des mondes économiques et scolaires distincts, ces différents savoirs, ensemble, ne vont pourtant pas sans incohérence. L'adéquation quantitative entre les "goûts et aptitudes » des élèves d'une part, les besoins en professions d'autre part, constitue notamment un angle mort. Loin de constituer une doctrine intégrée, ces savoirs posent des problèmes de recherche qui ont leur pendant dans les topoï de la réforme : le poids des structures socioprofessionnelles sur la scolarité des élèves, les enjeux économiques du système éducatif.

\section{Le dessin du système}

Comment ces dynamiques et perspectives propres aux mondes du travail et de l'enseignement composent-elles une politique scolaire ? Le comité créé en décembre 1954 pour proposer un avant-projet de réforme au ministre de l'Éducation nationale, Jean Berthoin, permet de l'observer. Ce comité réunit en effet les différentes catégories d'acteurs rencontrées jusqu'ici : directeurs d'administration centrale, psychologues, pédagogues, experts en prévision économique et représentants du monde de l'entreprise.

Solliciter des «personnalités compétentes, non plus seulement sur le plan universitaire, mais sur le plan de la vie française ${ }^{96}$ " pour définir la réforme de l'enseignement est une innovation revendiquée d'emblée ${ }^{97}$. Ces experts ont

\footnotetext{
95. Reuchlin (M.), «L'état des connaissances et la candidature à l'entrée en 6 " , BINOP, 14, 1958.

96. Citation de J. Berthoin au cours des débats parlementaires, 12-13 décembre 1954, AN F ${ }^{17} 17506$.

97. Cf. les correspondances de J. Berthoin en 1954 et 1958, AN F ${ }^{17} 17506$ et 17509.
} 
vocation à circonvenir les rivalités entre les ordres d'enseignement. Pour formuler les « besoins » auxquels l'enseignement doit s'adapter, ils ne connaissent pas de contradicteur : au cours des dix-neuf réunions hebdomadaires, aucun directeur ne discute leurs affirmations sur ce terrain, aussitôt acceptées qu'exposées. Ce comité marque une rupture : le gouvernement n'y revendique pas un projet politique, mais la capacité d'identifier et de répondre à des besoins et attentes ; l'action publique dirige moins la population qu'elle ne se présente à son service ; l'éducation n'est pas instituante, mais fonctionnelle; cette fonctionnalité se rapporte non à des politiques, mais à la population.

Or, en pratique, le comité répond non pas à des attentes sociales, mais à des besoins en matière économique. Trois des porte-parole « de la vie française » sont liés aux prévisions économiques : A. Sauvy et J. Fourastié, entrepreneurs de leur développement scientifique, et Jean Vergeot, l'un de leurs principaux soutiens et usagers en tant que commissaire adjoint du Plan. Trois autres de ces experts sont chefs d'entreprise : outre le président d'une société nationalisée, Émile Bollaert, figurent deux membres du CNPF : Raymond Boulenger ${ }^{98}$ et Jean Louis ${ }^{99}$. Vivement sollicité, le responsable de la SNCF, Louis Armand, décline faute de temps ${ }^{100}$. Ni économistes ni industriels, les deux autres membres extérieurs à l'administration de l'enseignement Charles Morazé et Robert Debré se montrent au cours des débats, eux aussi, très proches des idées des économistes. Ce privilège donné aux représentants de l'économie n'est sans doute pas sans lien avec la politique gouvernementale mendésienne, qui reconnaît un rôle à la formation professionnelle ${ }^{101}$. Mais, il n'est pas non plus dissociable des dynamiques institutionnelles propres à l'Éducation nationale. Car ces experts, prévisionnistes de l'emploi, sont ceux en qui Charles Brunold avait trouvé, un an plus tôt, la ressource pour porter sa réforme. Or c'est ce même Ch. Brunold qui souffle à son ministre cette procédure originale ${ }^{102}$ et insiste, au cours des débats, sur la primauté de leur opinion en cas de conflit.

\footnotetext{
98. Membre du comité directeur du CNPF depuis 1951 au moins, président de la commission pour l'apprentissage et la formation professionnelle en 1955, R. Boulenger préside le groupement des patrons de la bijouterie aux échelons parisien et national. Annuaire général du patronat français, Paris, CNPF, 1951, p. $64-65$ et 1955 , p. $70-71$.

99. En 1955, J. Louis dirige la société Babcock et Wilcox qui produit des équipements industriels (e.g. chaudières, citernes). Il est également vice-président du Syndicat général des industries mécaniques et transformatrices des métaux (Annuaire du CNPF 1951, p. 380, et 1955, p. 427).

100. Il accepte cependant de participer ponctuellement au comité pour y présenter un exposé. Son nom figure cependant sur la liste régulière des membres du comité, non sans appuyer la mise en scène d'un comité ouvert aux agents extérieurs à l'Éducation nationale.

101. Cet aspect peut être nuancé en constatant que le privilège aux représentants de l'économie sera interprété au cours des années 1960 comme fruit spécifique du régime gaullien. Cf. les articles de la publication du Parti communiste français L'école et la nation pendant cette période ainsi que les monographies de Loi (M.), Le désastre scolaire, Paris, Éditions sociales, 1962, et Segré (M.), École, formation, contradictions. De la réforme Berthoin-Fouchet à la réforme Haby, Paris, Éditions sociales, 1976.

102. Lettre de Ch. Brunold à J. Berthoin, 2 décembre 1954, AN CHAN F ${ }^{17} 17506$.
} 
Le fonctionnement de la commission et son projet de réforme montrent sur quelles formes institutionnelles les acteurs de l'enseignement public s'accordent avec les porte-parole de l'économie. D'une part, ils ne posent plus le problème en termes de catégories d'établissements (qui, renvoyant sans ambiguïté à des tutelles administratives et catégories d'enseignants, imposent de prendre parti dans leurs rivalités) mais en termes d'enseignements (ce qui permet d'euphémiser et d'écarter la question des établissements et tutelles qui les mettent en place). Ces enseignements donnent des entités abstraites et commensurables qui permettent de raisonner comme si les élèves pouvaient s'y trouver répartis rationnellement. D'autre part, ces enseignements ne sont légitimes qu'au regard de leur adéquation à des emplois ${ }^{103}$. Si les experts ne sont pas d'accord sur les catégories de main-d'œuvre et, partant, d'enseignement à développer en priorité, ils défendent farouchement le principe suivant lequel tout diplôme doit certifier d'une préparation à l'entrée, au niveau correspondant, dans le monde du travail ${ }^{104}$. «L'enseignement moyen » illustre ce genre nouveau : d'une part, il élude la question de savoir qui va l'assurer; d'autre part, sa raison d'être n'est pas d'enseigner, mais d'orienter les élèves afin de les affecter conformément à l'intérêt de l'institution scolaire comme à celui de l'économie. Les enseignements qui lui sont désormais reliés s'en trouvent bouleversés : ils n'accueillent plus des élèves venus fréquenter des filières données, mais des élèves orientés. L'intégration en système emporte ainsi une logique irréductible aux mouvements scolaires et professionnels qu'elle articule : elle n'est plus seulement une somme d'établissements qui offrent des programmes de formation à des curricula ou catégories d'emploi spécifiques (comme pourraient le souhaiter des directions administratives concentrées sur leur territoire spécifique ou des employeurs concentrés sur leur vivier de recrutement), mais une institution qui entend maîtriser des flux d'élèves.

Pour élaborer une offre publique d'enseignement agrégeant les différents points de vue en présence, le comité travaille à partir d'un schéma qui, sur un tableau noir ou une feuille de papier, aligne les établissements en un organigramme unique, et figure leurs liens avec l'économie par des flèches qui valent « débouchés ». Matériellement et formellement, ce schéma enregistre et nourrit l'interaction entre dynamiques internes à l'institution scolaire et pédagogisation

103. "Le projet de réforme devra fixer les niveaux de formation et, dans chaque niveau, diverses formes, adaptées aux exigences de la préparation aux emplois. Or l'avantage - inédit - du Comité institué par M. le Ministre est qu'il peut nous indiquer, grâce à sa composition, quels sont les niveaux et les formes d'enseignement qui sont nécessaires à l'économie et à la société contemporaines et qui, du même coup, assureront à nos élèves des emplois et des carrières authentiques. Ce n'est pas l'université elle-même qui peut déterminer ces exigences, mais ce sont les "utilisateurs" ", propos tenu par A. Le Gall - dont la posture de secrétaire général du comité consiste souvent à se faire la voix de son ministre - lors de la première réunion, AN CHAN F ${ }^{17} 17506$.

104. C'est à ce titre qu'ils demandent la suppression du baccalauréat, formation générale sans initiation professionnelle (cf. $14^{\mathrm{e}}$ et $15^{\mathrm{e}}$ réunions, 28 et 30 mars 1955). 
du travail. Il a également une portée performative en devenant le symbole de l'enseignement réformé. Ce schéma du système éducatif se retrouve notamment dans les brochures de propagande qui expliquent aux populations quel genre de bien la réforme de l'enseignement met à leur disposition : l'école, un ensemble d'établissements rationnellement organisés par lequel les élèves peuvent nourrir l'espoir d'accéder aux catégories d'emploi qui leur conviennent. Le système, un instrument d'action publique qui procède alors de l'interaction entre groupes sociaux aussi bien que de croyances individuelles, et par lequel une société se gouverne.

L'enseignement de la III ${ }^{e}$ République visait implicitement à reproduire les structures socio-économiques contemporaines. L'orientation de quelques élèves performants vers les filières d'élite restait une exception au sein d'une institution scolaire loin de constituer un levier politique pour un changement économique ${ }^{105}$. Pendant les "Trente glorieuses", au contraire, l'unification du système éducatif est explicitement conçue pour peser sur l'orientation des individus vers différentes catégories professionnelles - cadres et techniciens en particulier - qu'il s'agit de faire advenir. La réforme de 1959, héritière des travaux du comité d'étude pour la réforme de l'enseignement de 1954-1955, énonce ainsi l'impératif de produire les ressources humaines utiles à l'économie moderne :

« Nous ne pouvons plus maintenir une organisation scolaire qui ne nous permet de former qu'un chercheur, un ingénieur, un professeur quand il en faudrait deux, un technicien quand trois seraient nécessaires, tandis qu'à l'inverse, se presse dans nos enseignements supérieurs des lettres, de la philosophie et du droit une foule d'étudiants, à qui nous n’avions pas préparé d'autre issue, et qui doivent maintenant recourir à de tardives et difficiles reconversions, faute de quoi ils se condamneraient, et ce n'est pas sans exemple, à des besognes de fortune et de déboire ${ }^{106}$.»

Cette nouvelle perspective ne s'explique pas seulement par le changement de régime politique ou les idées politiques de nouvelles élites. Dès le début des années 1950, des mouvements de fond et des mobilisations propagent dans les mondes du travail l'intérêt pour la formation, tandis que l'Éducation nationale s'ouvre : l'intérêt des professionnels pour l'enseignement peut s'y projeter, l'institution scolaire se voit fonctionnalisée par des discours qui lui attribuent des finalités économiques et par de nouvelles formations qui s'ajustent aux «besoins » des professionnels. Dans ce contexte, le système éducatif qui se met

105. Lelièvre (C.), Nique (C.), La République n'éduquera plus, op. cit.

106. Exposé des motifs du décret portant réforme de l'enseignement public. 
progressivement en place agrège et recompose les enjeux de l'enseignement pour les différents secteurs professionnels. Le système éducatif devient un lieu où se déterminent les destins professionnels des élèves; c'est en se convertissant à ce jeu que les milieux populaires relaieront la fonctionnalisation de l'institution scolaire, et, partant, la scolarisation de la société française ${ }^{107}$ * $^{*}$

Philippe Bongrand est maître de conférences à I'Université de Cergy-Pontoise, chercheur au sein de l'équipe ÉMA (École, mutations, apprentissages, EA 4507). Ses recherches portent sur le processus par lequel I'institution scolaire noue et modèle action publique, savoirs, rapports sociaux et socialisation. II a récemment publié " L'introduction controversée de l'"excellence" dans la politique française d'éducation prioritaire (1999-2005) ", Revue française de pédagogie, 177, 2011.

philippe.bongrand@u-cergy.fr

107. Poullaouëc (T.), Le diplôme, arme des faibles. Les familles ouvrières et l'école, Paris, La Dispute, 2010.

* Merci aux membres du CURAPP qui ont accompagné cette recherche et aux relecteurs de la revue, ainsi qu'à Sylvie Aebischer et Ludivine Balland, pour leur aide à l'écriture de ce texte. 